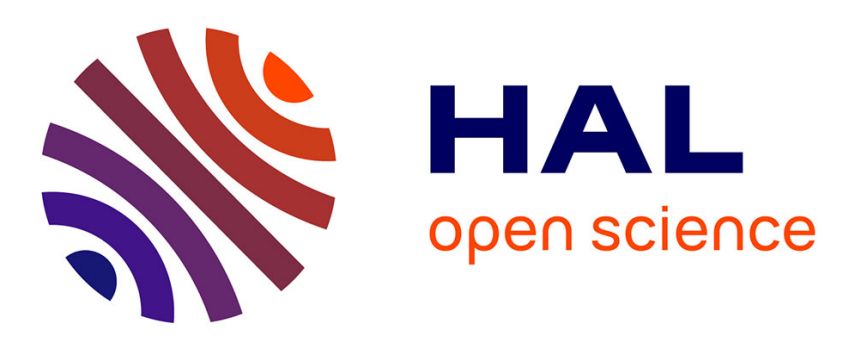

\title{
Using Image-based CFD to Investigate the Intracardiac Turbulence
}

\author{
Christophe Chnafa, Simon Mendez, Ramiro Moreno, Franck Nicoud
}

\section{To cite this version:}

Christophe Chnafa, Simon Mendez, Ramiro Moreno, Franck Nicoud. Using Image-based CFD to Investigate the Intracardiac Turbulence. Modeling the Heart and the Circulatory System, 14, Springer International Publishing, pp.97-117, 2015, MS\&A, 978-3-319-05230-4. 10.1007/978-3-319-05230-4_4 . hal-01152496

\section{HAL Id: hal-01152496 https://hal.science/hal-01152496}

Submitted on 18 May 2015

HAL is a multi-disciplinary open access archive for the deposit and dissemination of scientific research documents, whether they are published or not. The documents may come from teaching and research institutions in France or abroad, or from public or private research centers.
L'archive ouverte pluridisciplinaire HAL, est destinée au dépôt et à la diffusion de documents scientifiques de niveau recherche, publiés ou non, émanant des établissements d'enseignement et de recherche français ou étrangers, des laboratoires publics ou privés. 


\title{
Using image-based CFD to investigate the intracardiac turbulence
}

\author{
C. Chnafa, S. Mendez, R. Moreno and F. Nicoud
}

\begin{abstract}
A numerical framework designed to compute the blood flow in patientspecific human hearts is presented. The geometry of the heart cavities and associated wall motion are extracted from 4D medical images while the valves of the heart are accounted for thanks to low order geometrical models. The resulting blood flow equations are solved using a fourth-order low-dissipative finite-volume scheme and a mixed Aribtrary Lagrangian-Eulerian / Immersed Boundary framework. On top of retrieving the main fluid flow phenomena commonly observed in the left heart, the methodology allows studying the heart flow dynamics, including the turbulence characteristics and cycle-to-cycle variations.
\end{abstract}

\section{Introduction}

Heart pathologies are closely related to intracardiac hemodynamics. Recent technological innovations in imaging techniques have provided valuable opportunities for direct non-invasive in vivo assessment of hemodynamics. Blood flow velocities can be measured in vivo using phase-contrast magnetic resonance imaging (PC-MRI) or by echocardiography techniques.

PC-MRI studies have contributed to the understanding of the main hemodynamic features $[10,18,25]$. Although very comprehensive, the PC-MRI velocity mapping

C. Chnafa

I3M UMR 5149, University Montpellier II, FRANCE, e-mail: christophe.chnafa@univ-montp2.fr

S. Mendez

I3M, UMR 5149, University Montpellier II, FRANCE e-mail: simon.mendez@univ-montp2.fr

R. Moreno

Radiology Department, CHU of Rangueil, FRANCE e-mail: moreno.r@chu-toulouse.fr

F. Nicoud

I3M, UMR 5149, University Montpellier II, FRANCE e-mail: franck.nicoud@univ-montp2.fr 
is not real-time. Hence, beat-to-beat variations in the flow cannot be recorded (the $\mathrm{k}$-space is filled over many cardiac cycles). Moreover, PC-MRI suffers from a relatively low spatio-temporal resolution, precluding the observation of small scales and fast time-varying flow features [15].

Echocardiography techniques [11, 19], with higher spatio-temporal resolution make an alternative to PC-MRI. However, they only give access to velocity components directed towards or away from the ultrasonic beam, while one would want to measure the full 3D flow vectors. Nevertheless, investigations have been conducted on normal and abnormal hearts and interesting potential indicators of cardiac health hemodynamics arose $[4,13,16]$.

With the development of these cardiac imaging techniques, patient-specific geometries have been progressively used in computational fluid dynamics (CFD) $[5,21,28,30,42,44]$. Realistic heart wall movements on the basis of cine MRI or Computed Tomography (CT) scan data can be used: heart movement is prescribed from the patient-specific medical images, which can be acquired using standard clinical imaging procedures, instead of being computed. This strategy allows computation of the patient-specific hemodynamics and provides detailed insights into the cardiac flow field, providing potentially valuable clinical information. If the feasibility of this kind of approach has been shown, the results usually suffer from limited spatial resolution, partial geometry (only the left ventricle (LV) is considered in most cases) or numerical limitations (dissipative schemes). Besides, except in a few experimental works [7,41], cycle-to-cycle variations in the heart flow is an issue that is rarely dealt with.

In the present work, an image-based CFD method developed to compute flows in aortas [29] is extended to compute intracardiac flows. Medical images are used to generate a moving patient-specific domain, in which the blood flow equations are solved. Heart geometry movements are generated from a 4D sequence (MRI or CT scan images) treated by an appropriate image registration algorithm [35]. A specific attention is paid to the generation of a high-quality mesh which deforms consistently with the heart motion. This allows solving the flow equations with an essentially non-dissipative scheme compatible with the description of unsteady turbulent flows by Large Eddy Simulation. In order to demonstrate the ability of the method to compute heart flows, application to a complete human left heart described by ECG-gated 3D CT scan images is presented. The resulting flow is described, emphasizing the flow characteristics usually reported in the literature. Characteristics of the cycle-to-cycle variations are also reported.

The numerical method is detailed in $\S 2$. The characteristics of the applied case is presented in $\S 3$ and the flow field obtained is described in $\S 4$. Concluding remarks are given in $\S 5$. 


\section{Methodology}

The present computational method approaches couples an Arbitrary-Lagrangian Eulerian framework with an immersed boundary method in order to represent the blood flow within the moving endocardium while accounting for the heart valves motion. This section describes the flow solver and the treatment of the medical images needed to perform the computations.

\subsection{Fluid problem}

Blood is an incompressible, non-Newtonian fluid [9]. However, in large vessels, non-Newtonian effects are usually neglected in numerical simulations [30, 46] and constant kinematic viscosity $v$ is assumed in this paper. Note however that the present numerical method could be applied to non-Newtonian fluids.

The flow is thus governed by the incompressible Navier-Stokes equations (NSE), over a moving domain $\Omega_{f}(t) \subset \mathbf{R}^{3}$ of boundary $\partial \Omega_{f}(t)$. The Arbitrary Lagrangian Eulerian (ALE) framework [8] is used in order to account for the computational domain deformation over time. Introducing the pointwise computational domain velocity $\mathbf{u}_{\mathbf{g}}$, the NSE read:

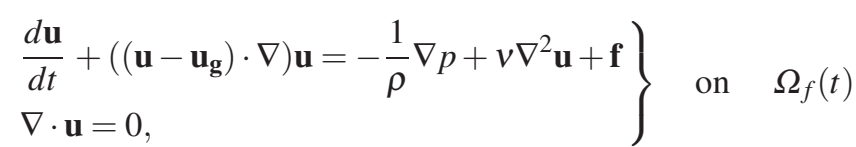

where $\mathbf{u}$ is the fluid velocity, $p$ is the pressure, $\rho$ the density and $\mathbf{f}$ a force per mass unit. Note that the time derivative denotes an ALE time derivative [8]. At the boundary of the computational domain, no-slip conditions are applied on walls $\left(\mathbf{u}=\mathbf{u}_{\mathbf{g}}\right.$ ) and Dirichlet conditions are applied over the inlet boundaries.

These equations are implemented in the flow solver YALES2BIO (www.math.univmontp2.fr/ yales2bio) [27], developed from the massively parallel finite-volume flow solver YALES2 [34]. At each time step, the grid velocity $\mathbf{u}_{\mathbf{g}}$ at each node of the computational domain is calculated (see next section). A projection method is used to solve the NSE: the momentum equation is first advanced using the fourthorder Runge-Kutta time-advancement scheme. Fluxes are discretized with a 4thorder central scheme. Any forcing term coming from immersed boundaries is accounted for in this prediction step. At the end of the prediction step, the grid reaches the final position of the time step. Hence, the projection step to calculate pressure is performed over a fixed grid. A Deflated Preconditioned Conjugate Gradient algorithm is used to solve the Poisson equation [24] involved in the projection step. Note that due to the transitional nature of the flow, Reynolds Averaged Navier-Stokes (RANS) approaches for modelling turbulence (e.g. $k-\varepsilon, k-\omega$ models, etc) are not appropriate since they rely on the assumption that turbulence is fully developed and ergodic. Even if adaptations have been proposed in order to handle transition, they 
essentially require the user to prescribe the transition location in advance. Instead, in the present study, the Large Eddy Simulation approach is followed, taking advantage of the low-dissipative scheme of integration used in YALES2BIO. In this view, only the smallest scales are modelled (scales smaller than the mesh size) while the evolution of the large scales is computed by solving a filtered version of NSE [33, 39, 43]. In the latter, a subgrid-scale model must be used in order to account for the effect of the unresolved scales on the dynamics of the resolved ones. This is usually done by an eddy-viscosity-based model [45]. In the present study, where the flow is strongly confined and piloted by the wall motions, an advanced subgrid scale model able to represent the proper turbulence damping near solid walls was used [36] as well as a well established formulation based on the dynamic procedure of Germano [12].

\subsection{Computational Domain}

\subsubsection{Extraction of the heart deformation}

Determining the movement of the computational domain, where the NSE are solved is all but an easy task. One solution is to compute the deformations of the boundary thanks to a full electrical-fluid-structure interaction solver. Unfortunately, there are many uncertainties regarding the constitutive laws (both mechanical and electrical) of the heart muscle as well as the external constraints the heart is submitted to. Moreover, accurate heart models are still under development today [47] as well as robust numerical method to solve them. A way of by-passing this issue is to extract the computational heart grid from 4D (3D + time) patient medical images. The following question must then be addressed: given several 3D images of a heart taken at different times in the heart cycle, how to extract the heart deformations from these images and how to deform a patient-specific grid accordingly? The first part of the question is actually a classical "image registration" problem.

Nowadays, there is a growing interest in the development of cardiac image registration methods [23]. Given two cardiac images, a template and a reference one, a transformation is determined to map the template image to the reference image. The deformation field can notably provide clinical information on the myocardial contractile function. Here, the same process is used to compute the heart deformations, but instead of focusing on functional data of the heart muscle, the computed deformation is used to extract patient-specific boundary conditions for the blood flow computation.

Among many registration algorithms [22, 31], voxel similarity measure techniques were preferred. This class of method operates directly on the image grey values, providing a flexible algorithm suitable for the complex heart movements.

In the present case, $N$ 3D images $I_{i}$ of a patient heart are taken at different times $t_{i}, 0 \leq t_{0}, t_{1}, \ldots, t_{N-1}<T$ during the heart cycle of period $T$. One of the $N$ images is selected as a template image. Note that this choice is somewhat arbitrary and that, without loss of generality, one can always tune the time origin so that the template 
corresponds to the $I_{0}$ image at time $t_{0}$. From this image, $N-1$ transformations $\psi_{i}$ are computed such that the transformed template image becomes similar to images $I_{i}(i$ between 1 and $N-1)$ successively considered as the reference image: transformation $\psi_{i}$ is search so that $I_{0}\left(\psi_{i}(\mathbf{x})\right)=I_{i}(\mathbf{x})$ for each voxel. Here, $I_{i}(\mathbf{x})$ stands for the voxel grey-level value at position $\mathbf{x}$ in the $i$-th $3 \mathrm{D}$ discrete image.

Each $\psi_{i}$ is calculated by minimizing the distance between $I_{0}\left(\psi_{i}(\mathbf{x})\right)$ and $I_{i}(\mathbf{x})$, an appropriate distance measure being based on the so-called sum of squared differences. The transformation $\psi_{i}$ is computed through an optimisation problem reading: given two images $I_{0}$ and $I_{i}$, find a mapping $\psi_{i}$ such that the squared intensity differences between $I_{0}\left(\psi_{i}(\mathbf{x})\right)$ and $I_{i}(\mathbf{x})$ is minimized for each voxel. The number of parameters describing the transformation is too high so that the solution $\psi_{i}$ of this problem is not unique. Additional constraints are needed to compute the mapping $\psi_{i}$.

In the present work, a constraint on $\psi_{i}$ is applied thanks to prior knowledge of the deformation sought [2]. The idea is to penalize unlikely deformations by imposing the heart deformations to be smooth. Bayesian statistics are used to obtain an $a$ posteriori computation of the deformation field. The prior deformation probability is incorporated through the Bayes' theorem: $p(\mathbf{Y} \mid \mathbf{I}) \propto p(\mathbf{I} \mid \mathbf{Y}) p(\mathbf{Y})$, where $p(\mathbf{I} \mid \mathbf{Y})$ is the likelihood of observing the images data $\mathbf{I}$ (template $I_{0}$ and reference $I_{i}$ images) given the deformation parameters $\mathbf{Y} . p(\mathbf{Y})$ is the prior knowledge of the deformation translated in the a priori probability of seeing the parameters $\mathbf{Y}$ and $p(\mathbf{Y} \mid \mathbf{I})$ is the a posteriori probability of getting $\mathbf{Y}$ knowing the two images data $\mathbf{I}$. Using this Bayesian framework, the goal is to maximise the probability $p(\mathbf{Y} \mid \mathbf{I})$. Knowing that a probability is related to its Gibbs form by $p(\mathbf{Y}) \propto e^{-H(\mathbf{Y})}$, the problem can be seen as a minimisation of the Gibbs potential:

$$
H(\mathbf{Y} \mid \mathbf{I})=H(\mathbf{I} \mid \mathbf{Y})+H(\mathbf{Y})+c,
$$

where $c$ is a constant. The likelihood potential $H(\mathbf{I} \mid \mathbf{Y})$ of observing the images data given the deformation parameters $\mathbf{Y}$ is directly linked to the squared intensity difference between $I_{0}\left(\psi_{i}(\mathbf{x})\right)$ and $I_{i}(\mathbf{x})$ for each voxel:

$$
H(\mathbf{I} \mid \mathbf{Y})=\frac{1}{2} \int_{\Omega}\left(I_{0}\left(\psi_{i}(\mathbf{x})\right)-I_{i}(\mathbf{x})\right)^{2} \mathrm{~d} \Omega,
$$

where the integral is taken over the image volume $\Omega$.

The second term $H(\mathbf{Y})$ in the right hand side of equation (2) is the wanted constraint, applied thanks to prior knowledge. The prior deformation knowledge put in this potential is expressed as a geometrical constraint on the mapping through the two studied images. A suitable prior probability is linked to the deformation of each voxel of the template image and to the reverse deformation [1]. This potential acting as a regularization term allows a penalization based on the Jacobian of the locals deformations $J$. A detailed description of this term can be found in [1].

This regularization term is weighted by a parameter $\lambda$ linked to the belief in the amount of deformation of the heart. A high value of the $\lambda$ parameter results in a high penalty on the voxels deformation, hence only small and smooth deformations are 
allowed. Given the high deformation of the left heart, high $\lambda$ values result in partial deformation and a high residual squared difference between the template image and the reference one. In the case of small values of $\lambda$, a lower residual squared difference will be reached, but the resulting deformation can be non physical due to excessive warping. Actually, the variability of heart movements is highly different from one region to the other. The aorta needs high values of $\lambda$, whereas small values are needed for the atrium or the ventricle.

One method of increasing the likelihood of achieving a good solution without introducing a spatial variability on $\lambda$ (and consequently avoiding more operatordependant work as well) is to apply successive filters to the images using a Gaussian smoothing convolution kernel $\mathscr{G}^{\sigma}$ of width $\sigma$. High-frequency information of the image is removed thanks to this filter then gradually re-introduced as the kernel width $\sigma$ becomes smaller and smaller. This iterative process is defined as the outer iterations: for each kernel width, distance between the images are minimized. This "coarse-to-fine" strategy has the effect of making the registration algorithm estimate the most global deformations during the first outer iteration, leaving out fine-scale structures. The optimum transformation for this kernel width is used to initialize the computation of the next optimum computation, which deals with finer details. This method increases the likelihood of finding the globally optimal match while avoiding the classical problem of the intensity-based method: their susceptibility to poor starting estimates.

As the regularization term, the intensity difference (first term $H(\mathbf{I} \mid \mathbf{Y})$ in the right hand side of the equation (2)) is weighted. The weight for the $j$-th inner iteration is defined as the inverse of the residual sum of the squared differences computed at the previous inner iteration of a given outer iteration of the algorithm and is denoted by $\frac{1}{d^{j-1}}$. Because $d$ has a high value for the first iterations, more weight is given to the regularization term, in order to get smooth deformations. As the algorithm gets close to the final solution, $d$ theoretically tends to zero, giving less weight to the priors and letting the algorithm computing more detailed deformations.

Finally, the sought transformation $\psi_{i}^{j, k}$ at the inner iteration $j$ and the outer iteration $k$ minimizes the function $f^{j, k}$ defined as:

$$
\begin{aligned}
f_{1}^{j, k}\left(\psi_{i}^{j, k}\right) & =\frac{1}{2 d^{j-1}} \int_{\Omega}\left(\left[\mathscr{G}^{\sigma^{k}} * I_{0}\right]\left(\psi_{i}^{j, k}(\mathbf{x})\right)-\left[\mathscr{G}^{j} \sigma^{k} I_{i}\right](\mathbf{x})\right)^{2} \mathrm{~d} \Omega, \\
f_{2}^{j, k}\left(\psi_{i}^{j, k}\right) & =g\left(J\left(\psi_{i}^{j, k}\right)\right), \\
f^{j, k}\left(\psi_{i}^{j, k}\right) & =f_{1}^{j, k}\left(\psi_{i}^{j, k}\right)+\lambda f_{2}^{j, k}\left(\psi_{i}^{j, k}\right),
\end{aligned}
$$

where the function $g$ is computed from the Jacobian singular values and determinant [2]. Finally the $N-1$ deformations are computed through this iterative optimisation process. This approach was successfully applied before to large vessels as the aorta cross [29]. 


\subsubsection{Patient-specific computational grid and application of the patient-specific deformation}

Once the $N-1$ mappings $\psi_{i}$ are computed, a patient-specific computational grid must be extracted from the template image and warped thanks to the computed deformations. The template image corresponding to volumetric data $I_{0}$ is imported into an image processing software (ScanIP;Simpleware Ltd., Exeter, UK). Before segmentation, the image $I_{0}$ is prepared: the region of interest is isolated and the image is smoothed to erase noise inherent to the medical images protocols. The segmentation itself is done by a classical thresholding method [38]. A suitable image intensity range which encompasses the voxel intensities of the region occupied by blood in the heart is selected. The quality of the segmentation relies on the operator's skills and knowledge of the human heart morphology and the quality of the chosen medical image. Image resolution plays a role in the potential domain simplification at this step. Trabeculae, left atrial appendage or papillary muscles can either be kept or neglected depending on the image quality and spatial resolution available. The 3D geometric reconstruction covers all the space occupied by blood in the left heart cavities. The surface of the geometric reconstruction of the heart is triangulated.

Once a template 3D patient-specific surface mesh is created, a procedure to deform this surface model thanks to the images must be provided. For each couple of images $\left(I_{0}, I_{i}\right)$ a suitable spatial transformation $\psi_{i}$ was found thanks to the method described in the previous section. These deformations $\psi_{i}$ are 3D deformation fields. Trilinear interpolation from these deformation fields to the template surface mesh is done. Thus, a set of $N-1$ successive surface meshes matching the physiological cardiac images at different times $t_{i}$ is produced as schematized in Fig. 1.

Position and velocity of all surface points are needed at any discrete time of the simulation, not only at the times $t_{0}, t_{1}, \ldots, t_{N-1}$. Since all the generated surface meshes share the same topology (number and connection between nodes, number of cells), interpolation is used to compute the position of each node and the velocity by taking the time derivative of this quantity. As geometry variations are periodic, a trigonometric interpolation is used. The surface position and velocities read:

$$
\left.\begin{array}{l}
\mathbf{x}_{s}(t)=\sum_{i=0}^{m}\left[a_{i} \cos \left(2 i \pi \frac{t}{T}\right)+b_{i} \sin \left(2 i \pi \frac{t}{T}\right)\right], \\
\mathbf{u}_{s}(t)=\frac{2 i \pi}{T} \sum_{i=1}^{m}\left[-a_{i} \sin \left(2 i \pi \frac{t}{T}\right)+b_{i} \cos \left(2 i \pi \frac{t}{T}\right)\right],
\end{array}\right\} \quad \text { on } \partial \Omega_{f}(t)
$$

where $T$ is the heart cycle period, $m$ the number of Fourier modes ( $m=\frac{N-1}{2}$ or $\frac{N}{2}$ depending on the parity of $N$ ) and $a_{i}, b_{i}$ the Fourier coefficients. Surface velocity $\mathbf{u}_{\mathbf{s}}$ needed at the computation domain boundary $\partial \Omega_{f}(t)$ is hence not computed as a FSI problem, but entirely extracted from the medical images. In the present study, it is used to handle the cardiac chambers and their connected vessels.

The template surface is imported in a commercial mesher (Gambit, ANSYS) to generate a template unstructured tetrahedral mesh. The computed boundary Fourier coefficients of Eq. (7) are interpolated in this template numerical domain. The com- 

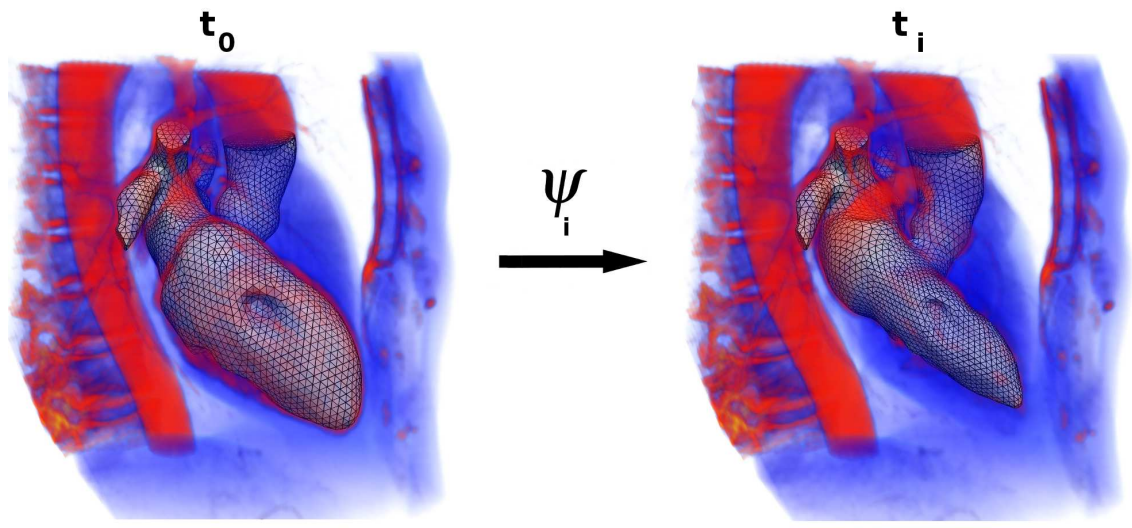

Fig. 1 Mesh deformation procedure applied to a left human heart. The template mesh segmented from the image at time $t_{0}$ is deformed thanks to $\psi_{i}$ to obtain the mesh at time $t_{i}$. This procedure is done for each image in the cardiac cycle in order to obtain the corresponding meshes.

putational mesh boundary now follows the shape of the patient endocardium and is updated in every step of the simulation. Motion of all internal points in the computational mesh is based on the prescribed boundary motion. At each iteration, nodal velocity $\mathbf{u}_{\mathbf{g}}$ is calculated through the computation of a Laplace equation [26] using the prescribed boundary motion as boundary condition for this problem.

The grid quality is monitored during the simulations. When the boundary displacement becomes too large compared to the local cell sizes, the cell quality can be highly deteriorated. This can lead to convergence problems or negative cell volumes. In this case, the numerical domain is re-meshed, Fourier coefficients are interpolated on this new discretized surface domain, as is the fluid solution at the new internal nodes.

\subsection{Valves}

Given their spatio-temporal resolution, MRI and CT scans fail to provide the necessary information to characterize precisely the movements of the aortic (AV) and of the mitral (MV) valves. Their geometry and movement have thus to be modelled. Accounting for the moving valves with the ALE method would be extremely complex due to grid quality issues. Here, an immersed boundary technique is used instead. A body force is imposed to drive the flow velocity to zero where the valves are located [3].

The opening and closing valve time is generally small (of the order of $5 \%$ of the heart cycle [48]). As a first modelling effort, it has been considered that valves open and close instantaneously, following the evolution of the ventricle volume. Systole lasts as long as the ventricle volume decreases. During systole, the AV is open and 
the MV closed. Diastole is defined as the heart period during which the ventricle volume increases: then, the AV is closed and the MV open.

Valve reconstruction starts with the definition of the valves annuli. Their geometry is reconstructed by inspecting the medical images. A number of markers are placed manually to define the valve annuli at a given time. The motion of these markers is then obtained thanks to the Fourier coefficient defining the boundary motion (Eq. 7).

As the focus is on the flow in the atrium and in the ventricle, the aortic valve is modelled very simply. Physiologically, the leaflets of the aortic valve are pushed against the vessel, offering small resistance to the flow. Hence, when the aortic valve is open, no immersed force is applied: aortic valve is only active when closed.

The mitral valve is represented by a more complex model, since its shape is expected to strongly impact the LV haemodynamics. The position of the mitral valve annulus being known over time, the mitral valve opening is defined using an ad hoc model. From visual inspection of the images, parameters as the average leaflets length, the orientation of the valve opening and an ellipse defining the opening area are imposed. Details of the procedure are described in Chnafa et al. [3].

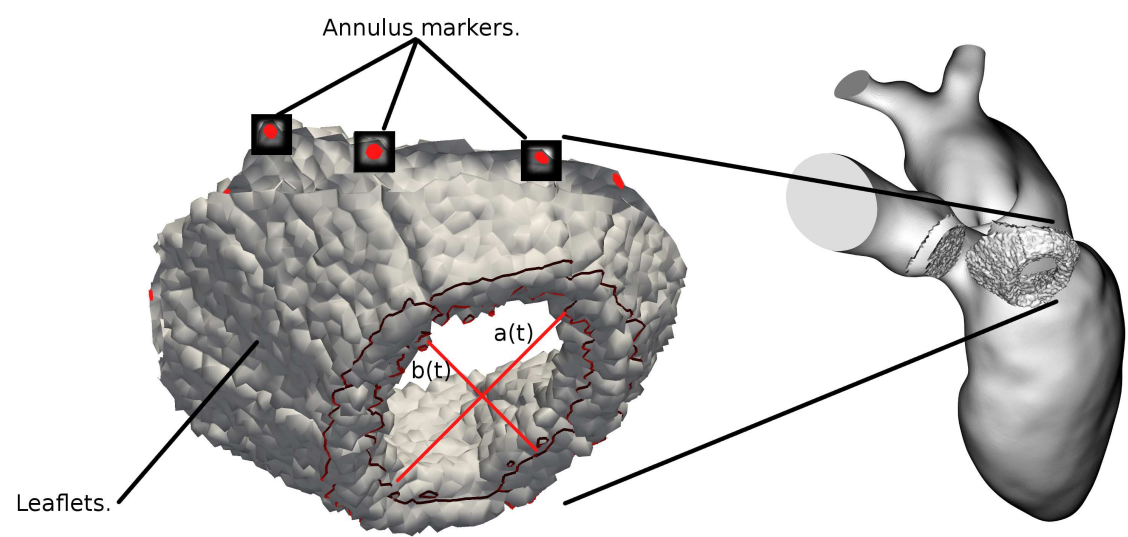

Fig. 2 Left plot: annulus markers and grid cells where a body force is added to model the MV leaflets. Diameters $a(t)$ and $b(t)$ of the elliptic opening are indicated. Right plot: full MV model included in the numerical domain (the AV is represented as well).

Knowing the MV leaflets position during the heart cycle, their effect on the blood flow is accounted for by using an immersed boundary method (IBM) [32]. For this purpose, the leaflets representations are given a thickness so that a few mesh nodes are located within the valves. Then, the force $\mathbf{f}$ in the NS equations (1) is set to impose the fluid velocity to zero within the leaflets. Figure 2 shows an example of the valves models during diastole, when the MV is open. 


\subsection{Inlet boundary conditions}

Inlet boundary conditions at the pulmonary veins must be provided. Under the present assumptions, either the aortic valve or the mitral valve is closed. Pulmonary veins thus always inject blood in a closed domain. As a consequence, blood being incompressible, the total mass flow rate entering the domain is entirely determined by the mass conservation constraint. With $Q_{P V}(t)$ denoting the inlet flow rate (sum of the volumetric flow rates at the four pulmonary veins) and $V_{L A}, V_{L V}$ and $V_{A O}$ denoting respectively the LA, LV and the aortic root volumes, mass conservation yields:

$$
Q_{P V}(t)= \begin{cases}\frac{d V_{L A}}{d t} & \text { during systole } \\ \frac{d V_{L A}}{d t}+\frac{d V_{L V}}{d t} & \text { during diastole. }\end{cases}
$$

Uniform velocity profile are imposed at each inlet condition. With $\mathbf{n}_{j}$ the outward normal vector and $A_{j}$ the area of inlet condition $j(j=1, . ., 4)$, the inflow velocity reads:

$$
\mathbf{u}_{j}(t)=-\left(\frac{Q_{i}(t)}{A_{j}} \zeta_{j}\right) \mathbf{n}_{j}
$$

where $\zeta_{j}$ determines the distribution of the flow between the four pulmonary veins. In the absence of additional measurement (typically flow rates from PC-MRI), it is fair to assume a uniform distribution between the different pulmonary veins $\left(\zeta_{j}=0.25\right.$ for all $j$.

\section{Application to a patient left heart: problem formulation}

\subsection{Heart model}

Using the framework described above, an actual CT exam of a patient treated at the University Hospital of Toulouse Rangueil (France) is used to generate a numerical domain and its deformation. The CT exam consists of $N=10$ medical images along the cardiac cycle of period $T=1 \mathrm{~s}$ with a spatial resolution $2 \times 2 \times 2 \mathrm{~mm}$.

Due to the limited resolution of the images, the intra-cardiac geometry is simplified. As shown in Fig. 3, the numerical domain includes the LA, LV, the aortic root and four pulmonary veins. Left atrial appendum and geometrical details as the cordae tendinae or papillary muscles are omitted. The LA has a height of $5.5 \mathrm{~cm}$ from the MV to the upper pulmonary root and a maximum diameter of order $4 \mathrm{~cm}$. The LV has a height of $8.8 \mathrm{~cm}$ from the MV to the apex (the lowest extremity of the LV in Fig. 3) and a maximum diameter of order $5 \mathrm{~cm}$. The four pulmonary veins 

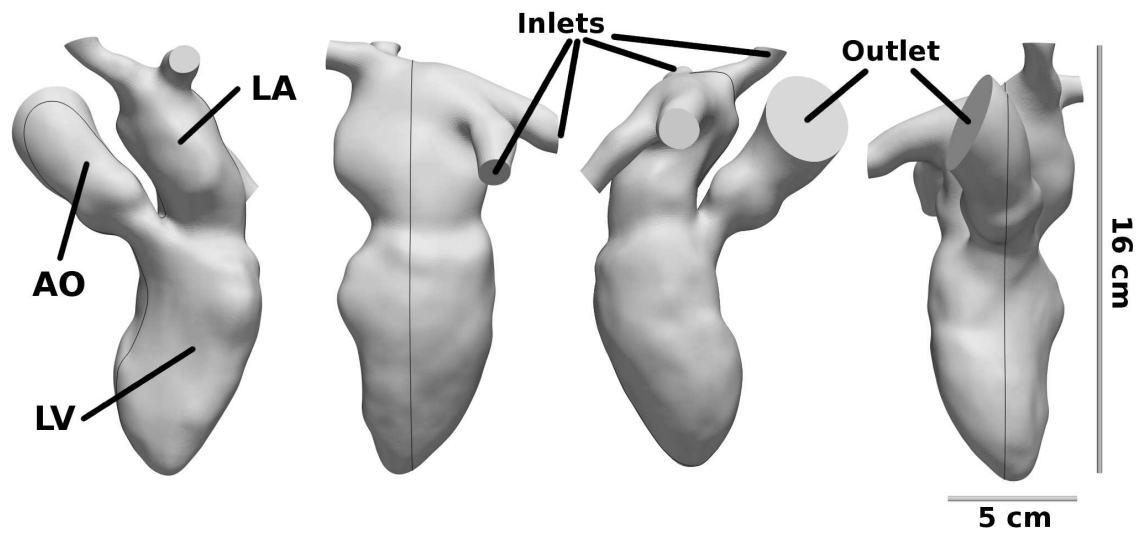

Fig. 3 Template computational domain extracted from a 3D medical image. The same domain is represented for four different points of view and the left ventricle (LV), left atrium (LA) and Aorta (AO) are indicated. Black line passing through the left heart indicates the position of slices used to describe the flow in section 4.

can be identified at the top of the views shown in Fig. 3. Each of the four pulmonary veins directly issues in the LA.

Valves are modeled as explained in section (2.3). A close examination of the medical images from the CT scan allowed to set the leaflets length to $l=12 \mathrm{~mm}$ for the MV. The open area presented to the blood flow is represented by an ellipse of axis $a=15 \mathrm{~mm}$ and $b=8 \mathrm{~mm}$. As a first approximation, this area is supposed constant over the time when the MV is open.

\subsection{Grid mesh and simulation details}

A nearly isotropic grid is created from the heart model described in the previous section using the commercial software Ansys Gambit, which was selected for its ability to generate good-quality tetrahedral mesh, appropriate for non-dissipative finite-volume formulations. The spatial resolution is of order $0.8 \mathrm{~mm}$, which yields grids of approximately three-million tetrahedral elements. In this study, in order to maintain the quality of the grid along the cycle, re-meshing (see section 2.2.2) was used at each acquisition instants $t_{i}, i=1, \ldots, N-1$.

The simulation time step is fixed by a CFL condition $(\mathrm{CFL}=0.9)$ consistent with the explicit time integration used in the CFD solver, which corresponds to a time step of order $10^{-4} \mathrm{~s}$.

Figure 4 displays the flow rates at the aortic valve (top plot), mitral valve (middle plot) and the heart inflow (bottom plot) gathering the four PV. Two verticals dotted lines separate the systolic phase and the diastolic one. For this heart, systole lasts $t / T=0.36$ (from $t / T 0.015$ to 0.375 ) and diastole $t / T=0.64$. 

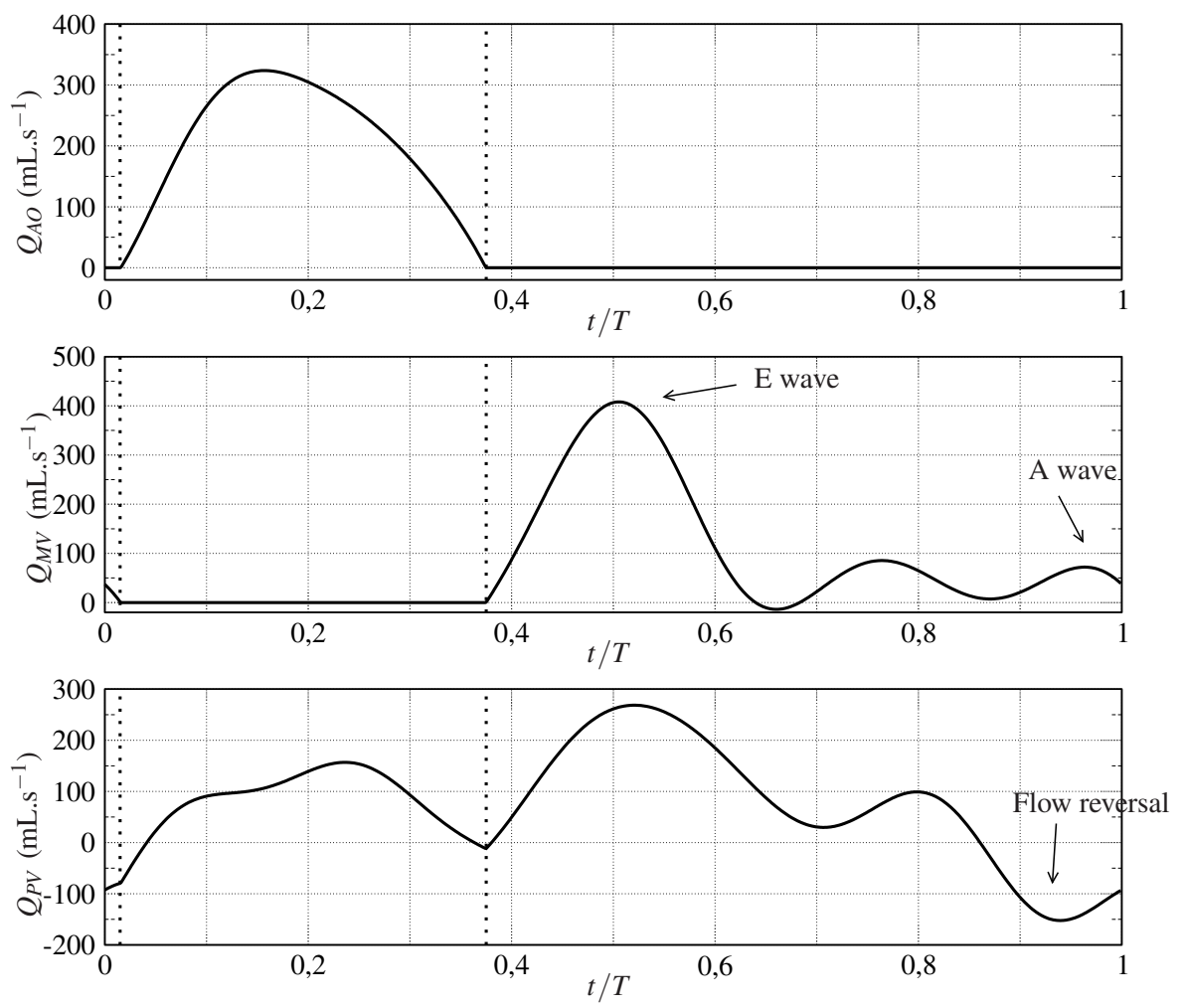

Fig. 4 Flow rates at the aortic valve (top plot), mitral valve (middle plot) and the total heart inflow (bottom plot) imposed at the pulmonary veins during the heart cycle. Vertical dotted lines mark the limit between the systolic phase $(t / T$ between 0.015 and 0.375$)$ and the diastolic phase.

$$
\begin{array}{ccccc}
\text { Element } & U_{\max } & D=2 \sqrt{\frac{A}{\pi}} \mathrm{Re}_{\max } & t_{m} / T \\
\text { AV } & 0.96 \mathrm{~m} . \mathrm{s}^{-1} & 2.20 \mathrm{~cm} & 5300 & 0.16 \\
\text { MV } & 1.08 \mathrm{~m} . \mathrm{s}^{-1} & 1.87 \mathrm{~cm} & 5000 & 0.52 \\
\text { Pulm. vein } & 0.79 \mathrm{~m} . \mathrm{s}^{-1} & 1.00 \mathrm{~cm} & 2000 & 0.52
\end{array}
$$

Table 1 Main flow parameters describing the simulation. The section-averaged maximum velocity is indicated as $U_{\max }$. Reynolds numbers are based on the diameter $D$ of the region of interest. The instants when they reach their maximum value $\mathrm{Re}_{\max }$ are reported as $\mathrm{t}_{m} / T$. For valves, $A$ is the area of the lumen when open. 
The pulmonary flow rate derived from time evolution of the heart cavities is consistent with classical medical dataThe aortic valve flow rate behaves as expected: it increases during systole with a maximum flow rate of $Q_{M V}=320 \mathrm{~mL} . \mathrm{s}^{-1}$, then decreases until its shutting at $t / T=0.375$. The aortic flow rate stays null during diastole. The mitral flow rate is usually composed by two peaks. The E wave, or rapid filling, and a second one, the A wave corresponding to late diastole. They are separated by a phase with almost no heart motion called diastasis. The flow rate shows only one main peak in the present case: the $\mathrm{E}$ wave $\left(t / T=0.51, Q_{M V}=410 \mathrm{~mL} \cdot \mathrm{s}^{-1}\right)$. The fact that the A wave is weak is symptomatic of pathologies.

The blood properties are: $\rho=1040 \mathrm{~kg} \cdot \mathrm{m}^{-3}$ and $v=4 \times 10^{-6} \mathrm{~m}^{2} \mathrm{~s}^{-1}$. Relevant flow conditions at different locations of the heart are reported in Table 1. Inlet Reynolds number for each pulmonary vein varies from 0 to approximately 2000, based on the vein diameter. The Reynolds number at the mitral tips varies from 0 to approximately 5000 (based on the effective mitral mean diameter $D=2 R_{a}=2 \sqrt{a b}$, the area of the open MV, the kinematic viscosity and the maximum flow rate). The maximum transmitral velocity $U_{\max }$ falls into the usual measurements (around 1.0 $\mathrm{m} . \mathrm{s}^{-1}$ ) [14]. The Reynolds number of the aortic valve is about 5300. Table 1 reports the maximum velocity magnitude and the maximum Reynolds number for different elements of the heart model: pulmonary veins, mitral valve and aortic valve. These ranges of Reynolds numbers and the pulsating nature of the inlet flow clearly indicate that this complex cyclic flow may be transitional if not fully turbulent. This justifies the use of Large-Eddy Simulation (LES) which is more suitable than other simulation approaches for unsteady and/or transitional flows. In order to achieve a quality assessment of the presented simulation, the Pope criterion [40] is used. According to this criterion, a reliable LES should be able to resolve at least $80 \%$ of the turbulent kinetic energy. Looking at the phase where the turbulent activity is the highest $(t / T=0.65)$, it was found that this criterion is met in $85 \%$ of the numerical domain. The last $15 \%$ are mainly located in the atrial cavity.

\section{Results and discussion}

\subsection{Global description of the cardiac cycle}

Fifteen cardiac cycles were simulated and phase-averaged in order to provide a clear view of the flow organization over the cardiac cycle. A detailed flow description can be found in [3]; only the main flow characteristics are recalled here for the sake of completeness. Six salient instants are illustrated in Figure 5: the ventricular mid and end-systole $(t / T=0.25-0.35)$, the beginning, peak and end of the $\mathrm{E}$ wave $(t / T=0.45-0.55-0.65)$ and the end of the A wave, just before the beginning of the next ventricular systole $(t / T=0.99)$. The velocity field is scaled by $u_{a}=\dot{q} l_{s} / V_{s}=0.1$ $\mathrm{ms}^{-1}$ where $\dot{q}=7.50 \times 10^{-5} \mathrm{~m}^{3} \mathrm{~s}^{-1}$ is the cardiac output, $V_{s}=5.55 \times 10^{-5} \mathrm{~m}^{3}$ is the end systolic volume and $l_{s}=7.40 \times 10^{-2} \mathrm{~m}$ is the ventricle length at the end of 

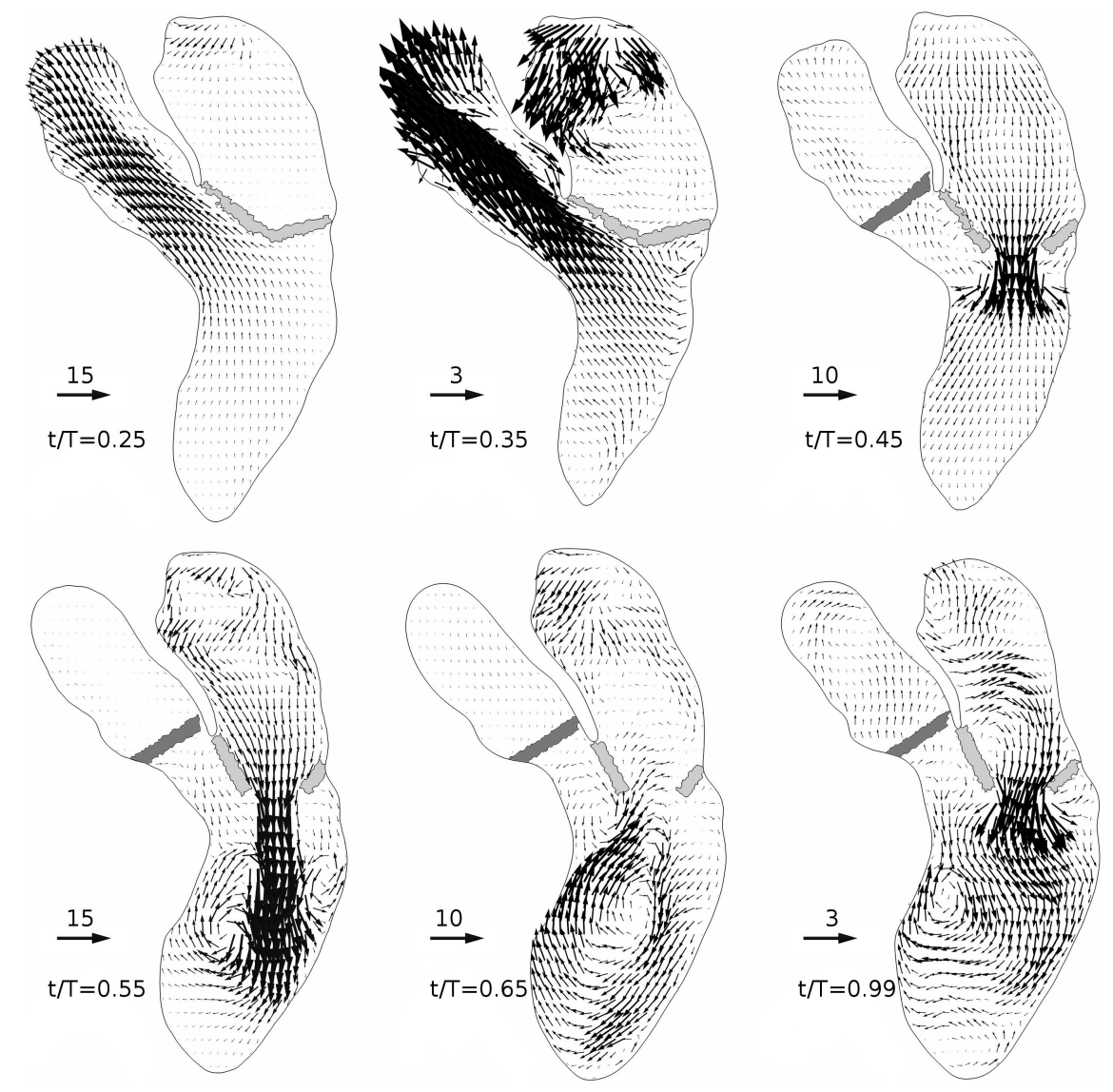

Fig. 5 Phase-averaged velocity field over a cutting-plane through the left heart. Velocity vector scale is not constant though the heart cycle and is indicated for each plot. Mitral valve is depicted in light grey the aortic one in dark grey.

systole. Note that due to the strong velocity variations along the cycle, the vector scale was adapted for each instant.

During systole $(t / T=0.25-0.35)$, the mitral valve is closed (in light gray in Fig. 5), preventing backflow towards the atrium, while the aortic valve is open (in dark gray in Fig. 5). The decrease of the ventricle volume causes ejection of blood into the aorta. Note that the velocity amplitude is the highest in the ascending aortic root. The computed flow at mid-systole is also highly swirled in the atrium, as reported in vivo $[18,25]$. This movement is hardly discernible in Fig. 5 , because the vortical movement is mainly perpendicular to the cutting plane [3]. Two recirculation zones are however visible in Fig. 5 (top center): just under the MV and within the aorta, just above the AV, in agreement with [17]. At the end of the ventricle contraction, the aortic valve closes and the mitral valve opens: ventricle filling starts. At $t / T=0.375$ 
(not shown), the ventricle diastole starts: the LV volume increases and blood passes from the LA to the LV, forming a strong jet through the MV. The shear layer between the jet generated during the $\mathrm{E}$ wave and the surrounding quiescent fluid rolls-up and shapes the jet head as a vortex ring [20]. The E wave vortex ring signature and its evolution are visible in Fig. $5(t / T=0.45-0.55)$ which illustrates a mechanism already reported by several authors [6]. The vortex ring does not remain symmetric, as the lateral wall prevents its full development. A large recirculating cell is clearly visible in the $\mathrm{LV}$ at $t / T=0.65$ (Fig. 5, bottom center), as often described in the literature $[16,21,25]$. It is characteristic of the flow in the ventricle after the $E$ wave. Two less intense blood recirculation zones can be detected: one at the apex, which is visible during the whole diastole and an intermittent one between the aortic valve and one of the MV leaflet. These blood recirculations are also described in silico [5, $30,44]$. Between the $\mathrm{E}$ wave and the A wave, the recirculating cell core in the LV moves from the ventricle center to the septum wall. During the A wave occurring at $t / T=0.99$ (Fig. 5, bottom right), the blood flux passing though the MV strengthens the recirculating cell in the LV, as classically reported [37]. Atrial contraction expels blood from the LA, both through the MV, as seen in the lower half part of the LA and through the pulmonary veins, as shown by the upward velocity vectors visible in the upper half part of the LA.

\subsection{Cycle-to-cycle variations}

The studied flow configuration is a breeding ground for weak turbulence. The transitional nature of this cyclic flow due to the highly complex evolving geometry and the unsteady inflows results in cycle-to-cycle variations. As an illustration, Figure 6 shows the time evolution of the vertical velocity at four different points within the left heart. Velocity signals corresponding to six different heart cycles are superimposed to visualize the cycle-to-cycle variations. Moderate $\left(2 u_{a}\right)$ to high $\left(5 u_{a}\right)$ variations are present at all the locations, although not uniformly distributed over the cardiac cycles. The second plot (corresponding to a probe located under the aortic valve) shows the lowest variations: almost no cycle-to-cycle variations are visible during the diastolic phase, which is expected, given the quiescent flow beneath the aortic valve during diastole. Cycle-to-cycle variations are however visible (variations around $2 u_{a}$ ) during the systolic phase, as expected given the typical Reynolds number. Cycle-to-cycle variations decrease during the systolic phase in the LV (see third plot), beginning from variations up to $4 u_{a}$ to almost null variations. This absence of fluctuations carries on even after the passage of E-wave vortex ring, visible on the signal. Variations are visible from $t / T=0.55$ and amplify during the late diastole. A similar behaviour is visible in the lower part of the LV (see fourth plot): during systole, variations decrease, then rise after $t / T=0.55$, reaching an amplitude of order $5 u_{a}$. The largest relative fluctuations are obtained in the left atrium (top plot) with small cycle-to-cycle variations during systole $(t / T$ between 0.015 and 0.375$)$ but fluctuations as large as $2 u_{a}$ at $t / T=0.35$ and $5 u_{a}$ at $t / T=0.6$. Theses variations 


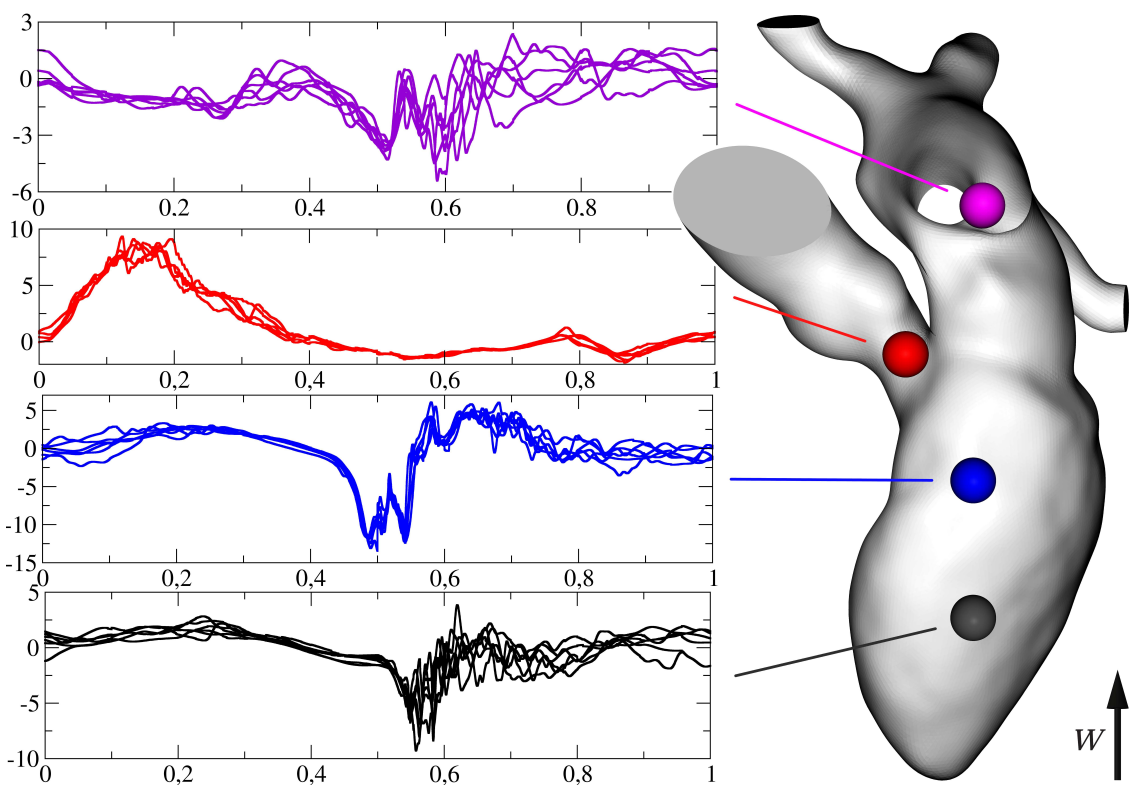

Fig. 6 Temporal evolutions of the scaled vertical velocity $w / u_{a}$ (the $w$ direction is indicated in the figure and $u_{a}=\dot{q} l_{s} / V_{s}$ ) at four different points within the left heart. Six cycles are reported to illustrate the cycle-to-cycle variations.

are related to the interaction between the four inlet flows from the four pulmonary veins.

A more quantitative assessment of the cycle-to-cycle variations is obtained by computing the kinetic energy of the velocity fluctuations. The velocity flow is decomposed in a mean (phase-averaged) velocity field $\bar{u}$ and a fluctuating part $u^{\prime}$. The turbulent kinetic energy $k$ and the mean flow kinetic energy $E_{k}$ are defined as:

$$
\begin{aligned}
k(t) & =\frac{1}{2 V(t)} \int_{V(t)}\left(u_{r m s}(\mathbf{x}, t)^{2}+v_{r m s}(\mathbf{x}, t)^{2}+w_{r m s}(\mathbf{x}, t)^{2}\right) \mathrm{d} V \\
E_{k}(t) & =\frac{1}{2 V(t)} \int_{V(t)}\left(\bar{u}(\mathbf{x}, t)^{2}+\bar{v}(\mathbf{x}, t)^{2}+\bar{w}(\mathbf{x}, t)^{2}\right) \mathrm{d} V
\end{aligned}
$$

where $u_{r m s}, v_{r m s}$ and $w_{r m s}$ are the root-mean-square values of the velocity fluctuations in the three directions. The volume $V(t)$ is either the volume of the ventricle or the volume of the atrium at time $t$.

Figure 7 shows how these energies evolve over the heart cycle, both in the ventricle (top plot) and the atrium (bottom plot). Note that 5 times $k$ is plotted, so that the same scale is used to represent both energies. The mean flow kinetic energy evolves similarly in both cavities. During the systolic phase $(t / T$ between 0.015 and 0.375$)$ it increases and reaches a plateau at $t / T=0.15$. After a decrease just before the beginning of diastole, the mean flow kinetic energy increases again and reaches a peak 

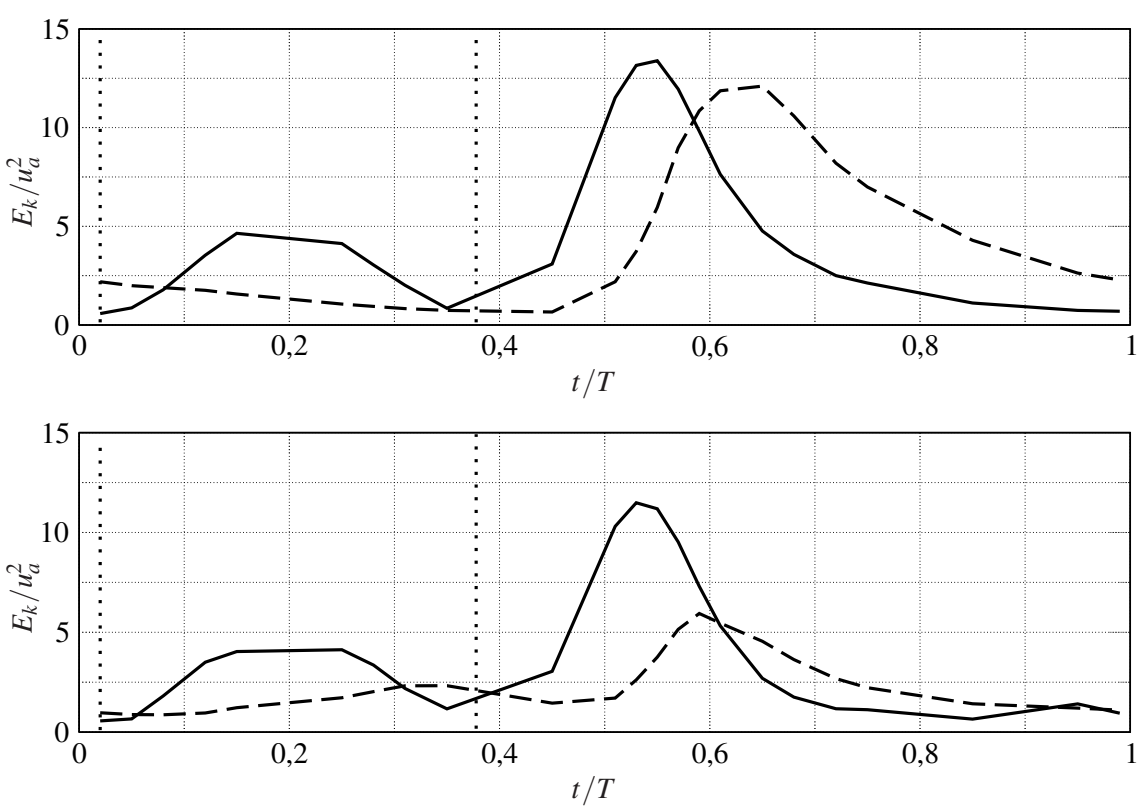

Fig. 7 Volumetric mean flow kinetic energy $\bar{E}_{k}$ (full line) and five times the turbulent kinetic energy $k$ (dashed line) in the left ventricle (top plot), and in the left atrium (bottom plot). The energies are nondimensionalised by $u_{a}^{2}$. Vertical dotted lines mark the limit between the systolic phase $(t / T$ between 0.015 and 0.375$)$ and the diastolic phase.

at $t / T=0.54$, viz. $0.04 T$ after the the peak of the $\mathrm{E}$ wave. The maximum value of $E_{k}$ corresponds to the presence of high velocities when blood flows from the atrium to the ventricle (see the mitral jet in Fig. 5, bottom left). The ventricular turbulent kinetic energy $k$ remains low during the systolic phase thanks to the stabilizing effect of the flow acceleration, with values of less than $5 \%$ of $E_{k}$. It then increases substantially, reflecting the amplification of the disturbance after the jet impingement on the lateral ventricle wall. The turbulent energetic peak is reached $T / 10$ after the peak of mean flow kinetic energy, corresponding to the convection time of the vortex ring and the decelerating phase of the flow. The turbulence intensity $k / E_{k}$ in the ventricle is as high as $50 \%$ during the $k$ peak.

The atrial turbulent kinetic energy behaves somewhat differently. First, it increases during the whole systolic phase, because of the interaction/collision of the four inflowing jets issued from the pulmonary veins. A first peak is thus reached near the beginning of the diastolic phase and turbulence intensity is then attenuated during the flow acceleration through the atrium, as expected. The atrial turbulent kinetic energy rises again after $t / T=0.5$ and reaches its peak before $t / T=0.6$ during the flow deceleration. On top of occurring earlier in the heart cycle, this peak is around twice less energetic than the one occurring in the ventricle. Still, it corresponds to a large turbulence intensity of approximatively $20 \%$. As in the ventricle, the turbulent kinetic energy then decreases until the end of the heart cycle. 


\section{Conclusion and outlook}

The approach presented here allows patient-specific blood flow simulations in the heart from a series of gated 3D images. Starting from 4D medical images, the numerical domain is first extracted and the heart wall movements are then calculated thanks to a proper image registration algorithm. In order to demonstrate the ability of the method to reproduce the cardiac flow, a computation of the blood flow in a whole left heart has been conducted. Results consistent with the current knowledge in terms of left heart flow is presented. All presented features have been reported several times in the literature, both in numerical and experimental studies and by medical imaging.

Furthermore, the use of fluid numerical method well adapted to fluctuating turbulent flows enables the observation of cycle-to-cycle variations in the flow field. Such variations are expected in the present flow, due to the high Reynolds numbers encountered and the unsteadiness of the flow incoming from the pulmonary veins. The present results show that in spite of rigorously identical contraction and boundary conditions, fluid inertia makes the flow differ from one cycle to another. More precisely, cycle-to-cycle variations in the left atrium can be observed in its upper part, where the collision of the jets issuing from the pulmonary veins makes the flow particularly chaotic. Spatially averaged kinetic turbulent energy level reaches a turbulent intensity of $20 \%$ at its peak then slowly decreases. In the left ventricle, velocity fluctuations are reported mainly during late diastole. Between the impact of the $\mathrm{E}$ wave jet on the lateral wall and the end of diastole, the left ventricle displays high levels of cycle-to-cycle fluctuations. Indeed, both the vortex ring impact and the E wave deceleration occur approximately at the same time, and both are features tending to generate turbulence. This translates into turbulent intensity levels as high as $50 \%$. This turbulent activity dissipates little by little, until the flow acceleration at early systole.

The presented method does not include or simplifies some aspects of the physiological heart. Blood is considered as a Newtonian fluid, which is an approximation commonly accepted for the heart flow. A non-Newtonian model could be included in our simulations. The spatio-temporal resolution of medical imaging imposes temporal interpolation and geometrical simplifications of the heart model. Consistently with the poor time resolution of the input medical data, a rough model of the mitral valve was used, which constitutes the main drawback of our method. Valves are instantaneously switch from their closed position to their open position and vice versa. This simple approach is justified by the fact that the opening and closing of the valves last only $5 \%$ of the whole cycle. Change of aperture area along time is not considered either. Note however that the model can be refined depending on the available data. Nevertheless, the presented approach allows to retrieve features reported in the literature and, in addition, it can provide detailed information about the complex intermittent flow in the left heart. 


\section{Acknowledgments}

The authors thanks Prof. D. Coisne and Dr. S. Nottin for interesting discussions about the presented results and their medical/physiological concordance. Dr. V. Moureau is gratefully acknowledged for giving access to the YALES2 solver.

\section{References}

1. Ashburner, J., Andersson, J.L.R., Friston, K.J.: High-dimensional image registration using symmetric priors. NeuroImage 9(6), 619-628 (1999)

2. Ashburner, J., Neelin, P., Collins, D.L., Evans, A., Friston, K.J.: Incorporating prior knowledge into image registration. NeuroImage 6(4), 344-352 (1997)

3. Chnafa, C., Mendez, S., Nicoud, F.: Image-based large-eddy simulation in a realistic left heart. Comp. Fluids 94, 173 - 187 (2014)

4. Cimino, S., Pedrizzetti, G., Tonti, G., Canali, E., Petronilli, V., De Luca, L., Iacoboni, C., Agati, L.: In vivo analysis of intraventricular fluid dynamics in healthy hearts. Eu. J. Mech. B/Fluids 35, 40-46 (2012)

5. Doenst, T., Spiegel, K., Reik, M., Markl, M., Hennig, J., Nitzsche, S., Beyersdorf, F., Oertel, H.: Fluid-dynamic modeling of the human left ventricle: Methodology and application to surgical ventricular reconstruction. Ann. Thor. Surg. 87, 1187-1195 (2009)

6. Domenichini, F., Pedrizzetti, G., Baccani, B.: Three-dimensional filling flow into a model left ventricle. J. Fluid Mech. 539, 179-198 (2005)

7. Falahatpisheh, A., Kheradvar, A.: High-speed particle image velocimetry to assess cardiac fluid dynamics in vitro: From performance to validation. Eu. J. Mech. - B/Fluids 35, 2-8 (2012)

8. Fernandez, M.A., Formaggia, L., Gerbeau, J.F., Quarteroni, A.: Cardiovascular Mathematics. Modeling and simulation of the circulatory system, Modeling, Simulation and Applications, vol. 1, chap. 3, The derivation of the equations for fluids and structure, pp. 77-121. Springer (2009)

9. Fung, Y.C.: Biomechanics - Circulation, 2nd edn. Springer-Verlag (1997)

10. Fyrenius, A., Wigstrom, L., Ebbers, T., Karlsson, M., Engvall, J., Bolger, A.: Three dimensional flow in the human left atrium. Heart 86, 448-455 (2001)

11. Garcia, D., del Alamo, J.C., Tanne, D., Yotti, R.L., Cortina, C., Bertrand, E., Antoranz, J.C., Perez-David, E., Rieu, R., Fernandez-Aviles, F., Bermejo, J.: Two-dimensional intraventricular flow mapping by digital processing conventional color-doppler echocardiography images. IEEE Trans. Med. Imag. 29, 1701-1713 (2010)

12. Germano, M., Piomelli, U., Moin, P., Cabot, W.H.: A dynamic subgrid-scale eddy viscosity model. Phys. Fluids 3, 1760-1765 (1991)

13. Gharib, M., Rambod, E., Kheradvar, A., Sahn, D., Dabiri, J.: Optimal vortex formation as an index of cardiac health. Proc. Natl. Acad. Sci. U.S.A. 103, 6305-6308 (2006)

14. Haugen, B.O., Berg, S., Brecke, K.M., Samstad, S.O., Slørdahl, S.A., Skjærpe, T., Torp, H.: Velocity profiles in mitral blood flow based on three-dimensional freehand colour flow imaging acquired at high frame rate. Eu. J. Echo. 1, 252-256 (2000)

15. Hollnagel, D.I., Summers, P.E., Poulikakos, D., Kollias, S.S.: Comparative velocity investigations in cerebral arteries and aneurysms: 3D phase-contrast MR angiography, laser doppler velocimetry and computational fluid dynamics. NMR in Biomed. 22, 795-808 (2009)

16. Hong, G.R., Pedrizzetti, G., Tonti, G., Li, P., Wei, Z., Kim, J., Baweja, A., Liu, S., Chung, N., Houle, H., Narula, J., Vannan, M.: Characterization and quantification of vortex flow in the human left ventricle by contrast echocardiography using vector particle image velocimetry. J. Am. Coll. Cardiol. 1, 705-717 (2008) 
17. Kilner, P., Yang, G., Mohiaddin, R., Firmin, D., Longmore, D.: Helical and retrograde secondary flow patterns in the aortic arch studied by three-directional magnetic resonance velocity mapping. Circulation 88, 2235-2247 (1993)

18. Kilner, P., Yang, G.Z., Wilkes, A., Mohiaddin, R.H., Firmin, D.N., Yacoub, M.H.: Asymmetric redirection of flow through the heart. Nature 404, 759-761 (2000)

19. Kim, H., Hertzberg, J., Shandas, R.: Development and validation of echo PIV. Expe. Fluids 36, 455-462 (2004)

20. Le, T.B., Sotiropoulos, F.: On the three-dimensional vortical structure of early diastolic flow in a patient-specific left ventricle. Eu. J. Mech. - B/Fluids 35, 20-24 (2012)

21. Long, Q.: Subject-specific computational simulation of left ventricular flow based on magnetic resonance imaging. Proceedings of the Institution of Mechanical Engineers, Part H 222, 475485 (2008)

22. Maintz, J.B.A., Viergever, M.A.: A survey of medical image registration. Medical Image Analysis 2(1), 1-36 (1998)

23. Makela, T., Clarysse, P., Sipila, O., Pauna, N., Pham, Q.C., Katila, T., Magnin, I.E.: A review of cardiac image registration methods. Medical Imaging, IEEE Transactions on 21(9), 1011$1021(2002)$

24. Malandin, M., Maheu, N., Moureau, V.: Optimization of the deflated conjugate gradient algorithm for the solving of elliptic equations on massively parallel machines. J. Comp. Phys. 238, 32-47 (2013)

25. Markl, M., Kilner, P., Ebbers, T.: Comprehensive 4D velocity mapping of the heart and great vessels by cardiovascular magnetic resonance. J. Cardiovasc. Magn. Reson. 13, 1-22 (2011)

26. Masud, A., Bhanabhagvanwala, M., Khurram, R.A.: An adaptive mesh rezoning scheme for moving boundary flows and fluid-structure interaction. Comp. Fluids 36(1), 77-91 (2007)

27. Mendez, S., Gibaud, E., Nicoud, .. An unstructured solver for simulations of deformable particles in flows at arbitrary reynolds numbers. JCP 256, 465-483 (2014)

28. Merrifield, R., Long, Q., Xu, X.Y., Kilner, P.J., Firmin, D.N., Yang, G.Z.: Combined CFD/MRI analysis of left ventricular flow. Med. Im. and augmented reality 3150, 229-236 (2004)

29. Midulla, M., Moreno, R., Baali, A., Chau, M., Negre-Salvayre, A., Nicoud, F., Pruvo, J.P., Haulon, S., Rousseau, H.: Haemodynamic imaging of thoracic stent-grafts by computational fluid dynamics (CFD): presentation of a patient-specific method combining magnetic resonance imaging and numerical simulations. Eu. Radiol. 22, 2094-2102 (2012)

30. Mihalef, V., Ionasec, R.I., Sharma, P., Georgescu, B., Voigt, I., Suehling, M., Comaniciu, D.: Patient-specific modelling of whole heart anatomy, dynamics and haemodynamics from fourdimensional cardiac CT images. Interface Focus 1, 286-296 (2011)

31. Modersitzki, J.: Numerical Methods for Image Registration (Numerical Mathematics and Scientific Computation). Oxford university press USA (2004)

32. Mohd-Yusof, J.: Combined immersed-boundary/B-spline methods for simulations of flow in complex geometries. Ann. Research Briefs, Center for Turbulence Research (1997)

33. Moin, P.: Advances in large eddy simulation methodology for complex flows. Int. J. Heat Fluid Flow 23(5), 710-720 (2002)

34. Moureau, V., Domingo, P., Vervisch, L.: Design of a massively parallel CFD code for complex geometries. Comptes Rendus Mécanique 339, 141-148 (2011). High Performance Computing

35. Nicoud, F., Moreno, R., Tayllamin, B., Chau, M., Rousseau, H.: Computational hemodynamics in moving geometries without solving the fluid-structure interaction problem. In: Conference on Modelling Fluid Flow (2009)

36. Nicoud, F., Toda, H.B., O. Cabrit, S.B., Lee, J.: Using singular values to build a subgrid-scale model for large eddy simulations. Phys. Fluids 23, 085,106 (2011)

37. Pedrizzetti, G., Domenichini, F.: Nature optimizes the swirling flow in the human left ventricle. Phys. Rev. Let. 95, 108,101 (2005)

38. Pham, D.L., Xu, C., Prince, J.L.: Current methods in medical image segmentation. Annu. Rev. Biomed. Eng. 2, 315-337 (2000)

39. Pope, S.B.: Turbulent flows. Cambridge university press (2000)

40. Pope, S.B.: Ten questions concerning the large-eddy simulation of turbulent flows. New J. Phys. 6(1), 35 (2004) 
41. Querzoli, G., Fortini, S., Cenedese, A.: Effect of the prosthetic mitral valve on vortex dynamics and turbulence of the left ventricular flow. Phys. Fluids 22, 041,901 (2010)

42. Saber, N.R., Wood, N.B., Gosman, A.B., Merrifield, R.D., Yang, G.Z., Charrier, C.L., Gatehouse, P.D., Firmin, D.N.: Progress towards patient-specific computational flow modeling of the left heart via combination of magnetic resonance imaging with computational fluid dynamics. Ann. Biomed. Eng. 31, 42-52 (2003)

43. Sagaut, P.: Large eddy simulation for incompressible flows, vol. 3. Springer Berlin (2000)

44. Schenkel, T., Malve, M., Reik, M., Markl, M., Jung, B., Oertel, H.: MRI-based CFD analysis of flow in a human left ventricle: Methodology and application to a healthy heart. Ann. Biomed. Eng. 37, 503-515 (2009)

45. Smagorinsky, J.: General circulation experiments with the primitive equations: 1. the basic experiment. Mon. Weather Rev. 91(3), 99-164 (1963)

46. Taylor, C.A., Figueroa, C.A.: Patient-specific modeling of cardiovascular mechanics. Annu. Rev. Biomed. Eng. 11, 109-134 (2009)

47. Vadakkumpadan, F., Arevalo, H., Ceritoglu, C., Miller, M., Trayanova, N.: Image-based estimation of ventricular fiber orientations for personalized modeling of cardiac electrophysiology. IEEE Trans. Med. Imag. 31, 1051-1060 (2012)

48. Yoganathan, A.P., He, Z., Casey, J.S.: Fluid mechanics of heart valves. Annu. Rev. Biomed. Eng. 6, 331-362 (2004) 